\title{
[Ni II] EMISSION IN SUPERNOVA REMNANTS
}

\author{
Richard B.C. Henry, University of Oklahoma \\ and
}

Robert A. Fesen, University of Colorado

\begin{abstract}
Combining new spectrophotometric data on the Orion and Crab Nebulae with detailed calculations, we show that strong [Ni II] $\lambda 7378$ emission observed in numerous SNR's may be associated with gas having $N_{e}>50,000 \mathrm{~cm}^{-3}$.
\end{abstract}

INTRODUCTION: Strong [Ni II] emission at $\lambda 7378$ in the Crab Nebula was first observed by Miller (1978). Subsequent spectrophotometric measurements by Péquignot and Dennefeld (1983) and Henry, MacAlpine, and Kirshner (1984) indicated that the strength of the $\lambda 7378$ line rivals that of $\mathrm{H} \beta$ throughout the entire filament system. Initial interpretations of these findings suggested that the ratio of nickel to iron exceeds its solar value by more than an order of magnitude (see Henry 1984a,b; Péquignot and Dennefeld 1983). Recently, observations by Dennefeld (1986) of two Galactic and three extragalactic supernova remnants indicated the presence of strong [Ni II] emission in these objects as well, leading again to inferred $\frac{N i}{F e}$ which is roughly an order of magnitude above solar. Such an abundance scenario would be compatible with supernova ejecta which contain large amounts of material synthesized under neutron-rich conditions, ie. deep inside the core of the precursor star. However, the overabundance interpretation is seriously challenged by the ubiquitous presence of relatively strong $[\mathrm{Ni}$ II] emission in objects where elemental enhancements are not expected, such as the SNR IC 443 (Fesen and Kirshner 1980), Seyfert galaxies (Halpern and Oke 1986), and Herbig-Haro objects (Brugel et al. 1981).

THE APPARENT NICKEL ABUNDANCE IN ORION: Henry (1984a,b) found an apparent abundance ratio of $\frac{N i}{\mathrm{Fe}}$ in Orion which was roughly an order of magnitude higher than the solar value, based upon observations by Grandi (1975) of [Ni II] $\lambda 7378$ and [Fe II] $\lambda 8617$ at one location in that Nebula. (The apparent abundance ratio refers to the value derived from a straightforward interpretation of line strengths, ignoring any unusual mechanisms which could alter the observed ratio.) In order to substantiate this conclusion, we have determined the apparent abundance of $\mathrm{Ni}$ at three additional locations in the Orion, this time with respect to $\mathrm{O}$, since the abundance of the latter is well established. We employed the Coudé feed telescope as well as the IIDS attached to the $2.1 \mathrm{~m}$ telescope, both at KPNO, to obtain spectrophotometric measurements of [Ni II] $\lambda 7378$ and [O II] $\lambda 7325$. The three locations correspond roughly to Peimbert and Torres-Peimbert's (1977) positions 1a, 3a, and 4a.

In order to determine the apparent $\frac{\mathrm{Ni}}{\mathrm{O}}$ abundance ratio at each of the positions, a general relation between the observed line ratio, $\frac{I(\lambda 7378)}{I(\lambda 7325)}$, and the number abundance ratio, $\frac{N i}{P}$, was established for $\mathrm{H}$ II regions by calculating numerous photoionization models over a range of stellar effective temperatures and total nebular densities. We employed the code recently used by Henry and Shipman (1986) but with the addition of nickel to the elements included in the calculations. Photoionization cross-sections for nickel were taken from Reilman and Manson (1979). 


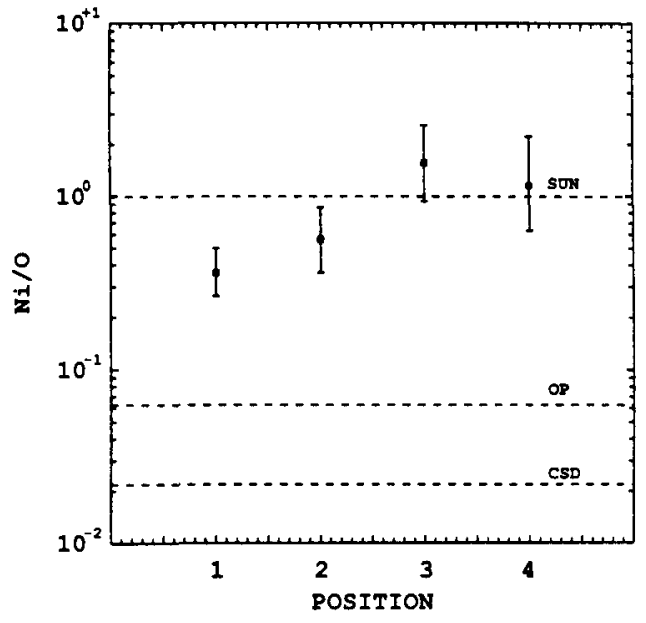

Fig. 1 - Ni/O number abundance ratio, normalized to the solar value, for four positions observed in Orion. Dashed lines toward the bottom are observed $\mathrm{Fe} / \mathrm{O}$ ratios (see text).

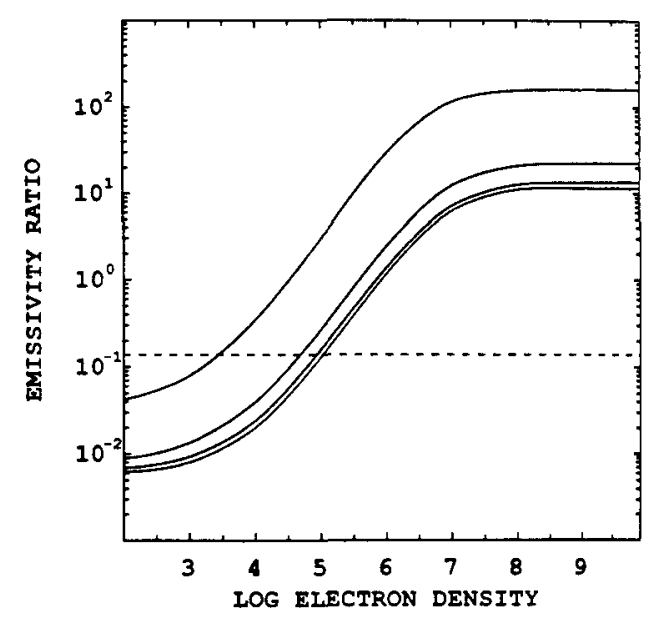

Fig. 2 - Calculated ratio of emissivities of $[\mathrm{Ni} \mathrm{II}] \lambda 7378 /[\mathrm{S} \mathrm{II}] \lambda 6724$, as a function of electron density for $1000 \mathrm{~K}$ (top curve), $5000 \mathrm{~K}, 10000 \mathrm{~K}$, and $15000 \mathrm{~K}$ respectively. The dashed line shows the mean observed ratio.

Since radiative and dielectronic recombination coefficients for nickel are not available in the literature, we employed the values for iron published by Woods, Shull, and Sarazin (1981). This is a reasonable substitute, due to the close similarities of the nickel and iron photoionization cross-sections, from which recombination cross-sections are calculated. In addition, we included a charge exchange rate for the $\mathrm{Ni}^{+2}+\mathrm{H}^{\circ} \rightarrow \mathrm{Ni}^{+}+\mathrm{H}^{+}$reaction calculated by Neufeld (1985). Finally, the collision strengths and transition probabilities for $\mathrm{Ni}^{+}$from Nussbaumer and Storey (1982) were used.

Using a relation derived in this way, we determined the apparent $\frac{N i}{O}$ ratio at each of our three positions plus that of Grandi (1975) using observed line strengths from our and Grandi's observations, as well as relevant data from Peimbert and

Torres- Peimbert (1977) which were necessary to determine the temperature and density at each of the locations. Our resulting abundance ratios are presented in Fig. 1, where we have plotted the $\frac{N i}{O}$ ratio, normalized to its solar value, for each position. The errors in the abundances include contributions from estimated observational uncertainties in line strengths, temperatures, densities, and the abundance-determining technique itself. The two dashed lines toward the bottom of the plot indicate the $\frac{N i}{O}$ levels which would be expected in Orion if $\mathrm{Ni}$ and Fe are depleted by the same fractions. The levels shown correspond to determinations of the Fe abundance by Cosmovici et al. (1980) and Olthoff and Pottasch (1975). We adopted the $O$ abundance of Peimbert and Torres-Peimbert (1977), who found $\mathrm{O}$ to be present at a level that was very nearly solar.

Our results indicate that the apparent nickel abundance in Orion is roughly the same as it is in the sun, ie. more than an order of magnitude greater than the expected level. This finding agrees closely with the much simpler analysis in Henry $(1984 a, b)$. Unfortunately, no other strong lines of [Ni II] are observable in the optical, UV, or near IR which would allow independent checks on this result. 
PRODUCTION OF [Ni II] $\lambda 7378$ EMISSION: The chemical properties of nickel and iron are very similar, due to the similarity of the electron structures of the two elements. In the absence of evidence to the contrary, we must conclude that nickel and iron form grains at roughly equal rates. Thus, the possibility that iron fractionates out of gas and into the solid phase more readily than nickel does seems an unlikely explanation for the apparent overabundance of gas-phase nickel. We therefore proceed under the assumption that the apparent nickel excess is the result of either: 1) an enlarged $\mathrm{Ni}^{+}$zone in the nebula; 2) an excitation process other than pure inelastic collisions; 3) erroneous atomic data; or 4) unusual temperature or electron density conditions which favor $\lambda 7378$ production.

An enlargement of the $\mathrm{Ni}^{+}$zone would be produced if: 1) the photoionization cross-section of $\mathrm{Ni}^{\circ}$ were larger; 2) the total recombination rate onto $\mathrm{Ni}^{+}$were larger; or 3 ) the total recombination rate onto $\mathrm{Ni}^{\circ}$ were smaller. (The total recombination rate refers to the sum of radiative, dielectronic, and charge exchange recombination rates.) In any of the three cases, the effect is to increase the relative amount of $\mathrm{Ni}^{+}$at the expense of other ions of nickel. Since we are searching for order-of-magnitude effects, in order to enlarge the $\mathrm{Ni}^{+}$zone sufficiently, we must presume that the photoionization or recombination cross-sections are in error by an order of magnitude. For charge exchange, the coefficients calculated by Neufeld (1985) would have to be underestimated by a similar amount. On the other hand, the emissivity of $\lambda 7378$ could be increased above inelastic collision levels by excitation of the line through recombination. At the same time, order of magnitude errors in the published values for collision strengths and transition probabilities would result in an underestimation of $\lambda 7378$ emissivity. Finally, photon pumping mechanisms appear unimportant, since none of the excitation energies from the ground state of $[\mathrm{Ni} \mathrm{II}]$ coincide with strong nebular lines.

To test the effects of many of the above processes, we began by calculating a standard photoionization model for the Crab Nebula filaments which roughly fit the average line strengths observed by Davidson (1987) and Fesen and Kirshner (1982). We employed the solar ratio by number of $\frac{N_{2}}{H}$. This model produced a strength of $\lambda 7378$ which was more than a factor of 15 below observations. Next, we experimented with large changes in the relevant cross-sections, rate coefficients, or other atomic data discussed above and noted the effects such changes had on the relative strength of $\lambda 7378$. All experiments failed to uncover a process which is capable of explaining the strong observed $\lambda 7378$ emission in the Crab.

We then abandoned the use of models and considered physical conditions under which [Ni II] $\lambda 7378$ emissivity, $j_{\lambda 7378}$, would be expected to be high with respect to lines formed in the same region of the nebula by ions of more abundant elements. In particular, considerations of ionization potentials as well as ionization structure information gleaned from our photoionization models indicated that $\lambda 7378$ should be produced coincidentally with [S II] $\lambda 6724$. Therefore, we calculated the expected ratio of emissivities for a solar value of $\frac{N_{i}}{\mathrm{~S}}$ over a large range of temperatures and densities. Our results are shown in Fig. 2, where the dashed horizontal line indicates the mean observed value of the ratio of these two lines. From observational and model results, we expect the electron temperature of the gas to be between $5000 \mathrm{~K}$ and $10,000 \mathrm{~K}$. Therefore, observations of $\frac{I(\lambda 7378)}{I(\lambda 6724)}$ in the Crab imply densities between $10^{4.7}$ and $10^{5}$. These densities exceed the critical density of $\lambda 6724\left(\sim 10^{4} \mathrm{~cm}^{-3}\right)$ but are below that of $\lambda 7378,\left(\sim 10^{7} \mathrm{~cm}^{-3}\right)$, and thus under such conditions [Ni II] $\lambda 7378$ is produced more efficiently. However, since [S II] densities observed in the Crab are normally $\sim 10^{3} \mathrm{~cm}^{-3}$, we speculate that the low-ionization regions of the filaments may actually be composed of two 
components: a low density component $\left(N_{e} \sim 10^{3} \mathrm{~cm}^{-3}\right)$ which produces most of the [S II] emission, and a second component with a density exceeding $10^{4.7} \mathrm{~cm}^{-3}$ which produces the $[\mathrm{Ni}$ II] emission.

Certain observations will help to clarify this problem somewhat. We have attempted measurements of other optical lines of [Ni II] such as $\lambda 4326, \lambda 3999$, and $\lambda 6666$ in an effort to check results against predicted ratios of these lines to $\lambda 7378$ for verification that the observed emission in the latter is due primarily to collisional excitation. However, because of the weakness of these lines, definite conclusions cannot be drawn at the present time. Of considerable value for helping to confirm the excitation mechanism of $\lambda 7378$ would be the measurement of the strengths of [Ni II] lines at $1.19 \mu, 6.63 \mu$, and $10.7 \mu$, lines which are predicted by Henry (1987) to rival the strength of $\lambda 7378$. In addition, Nussbaumer and Storey (1982) have shown that $\frac{I(\lambda 7412)}{I(\lambda 7378)}$ is an effective temperature diagnostic, and Henry's (1987) results imply that $\frac{I(1.19 \mu)}{I(\lambda 7378)}$ is a particularly good probe of electron density. Thus observations of these ratios could help to establish the conditions in the gas that is responsible for the strong [Ni II] emission observed in the Crab Nebula and other SNRs.

\section{REFERENCES}

Brugel, E.W., Böhm, K.H., and Mannery, E. 1981, Ap.J. Suppl., 47, 117.

Cosmovici, C.B., Strafella, F., and Dirscherl, R. 1980, Ap.J., 236, 498.

Davidson, K. 1987, preprint.

Dennefeld, M. 1986, Astr. Ap., 157, 267.

Fesen, R.A., and Kirshner, R.P. 1980, Ap.J., 242, 1023. . 1982, Ap.J., 258, 1.

Halpern, J.P., and Oke, J.B. 1986, Ap.J., 301, 753.

Grandi, S.A. 1975, Ap.J. Letters, 199, L43.

Henry, R.B.C. 1984a, in Stellar Nucleosynthesis, ed. C. Chiosi and A. Renzini (Dordrecht: Reidel), p. 43. 1984b, Ap.J., 281, 644. 1987, Ap.J., 322, in press.

Henry, R.B.C., MacAlpine, G.M., and Kirshner, R.P. 1984, Ap.J., 278, 619. Henry, R.B.C., and Shipman, H.L. 1986, Ap.J., 311, 774.

Miller, J.S. 1978, A p.J., 220. 490.

Neufeld, D.A. 1985, private communication.

Nussbaumer, H., and Storey, P.J. 1982, Astr. Ap., 110, 295.

Olthof, H., and Pottasch, S.R. 1975, Astr. Ap., 43, 291.

Peimbert, M., and Torres-Peimbert, S. 1977, M.N.R.A.S., 179, 217.

Péquignot, D., and Dennefeld, M. 1983, Astr. Ap., 120, 249.

Reilman, R.F., and Manson, S.T. 1979, Ap.J. Suppl., 40, 815.

Woods, D.T., Shull, J.M., and Sarazin, C.L. 1981, Ap.J., $249,399$. 